\title{
Sclera Pattern Recognition for Identification
}

\author{
Rohan Parab \\ U.G. student \\ Dwarkadas J.Sanghvi College \\ of Engineering \\ Vileparle-Mumbai
}

\author{
Revathi A. S. \\ Assistant Professor \\ Dwarkadas J.Sanghvi College \\ of Engineering \\ Vileparle-Mumbai
}

\author{
Preeti Jha \\ U.G. student \\ Dwarkadas J.Sanghvi College \\ of Engineering \\ Vileparle-Mumbai
}

\begin{abstract}
In this paper a delineate the new human identification method is proposed: sclera recognition technique. Due to the uniqueness of the sclera pattern, it can be used as identification in place of code, fingerprint, face recognition and voice recognition. To distinguish different patterns, some tonal and illumination corrections are performed to get a clear sclera area without disturbing the vessel pattern structure[8]. This paper aims at developing a new method for sclera segmentation which works for both color as well as grayscale images. The blood vessel structure of sclera is different for different people and it lies in the region of the visible wavelengths, therefore it can be used for the human identification method (ID). To obtain shape and structure of a sclera vessel kernel functions are used in order to separate out the magnitude and phase plots. Gabor wavelet filter is a bidimensional Gaussian function which separates the $R$ \& $G$ plane of the scanned image and due to its $2 \mathrm{D}$ nature, the $\mathrm{B}$ plane is difficult to plot as well as recognize (mathworks). Also lot of people has blue iris which is difficult to identify, hence plotting the graph of $\mathrm{R}$ and $\mathrm{G}$ only would be easy.
\end{abstract}

\section{General Terms}

Image Processing, Matlab, Colormap

\section{Keywords}

Sclera Recognition, Gabor-wavelet filter, Kernel Function

\section{INTRODUCTION}

Researchers have worked out on a numerous techniques to keep the secrecy of their identity. These techniques include password protection, fingerprint scanning, facial recognition as well as voice recognition, etc but due to certain drawbacks in each, there is a high possibility that the personal identity could be revealed. This is why sclera recognition can prove to be a safe and sound method. In the traditional methods, the security-code can be easily determined by human errors, fingerprint of a person can be obtained from their imprints and voice can be manipulated to produce flaws in the systems. Sclera recognition can achieve comparable recognition accuracy to iris recognition in the visible wavelengths. Sclera blood veins have been investigated recently as a biometric trait which can be used in a recognition system. Sclera is the white and opaque outer protective part of the eye.This part of the eye has visible blood veins which are randomly distributed. This feature makes these blood veins a promising factor for eye recognition. The sclera has an advantage in that it can be captured using a visible- wavelength camera[2].
Therefore, applications which may involve the sclera are wide ranging. First, a colour-based sclera region estimation scheme for sclera segmentation has been developed. Thus the sclera portion of the human eye has been extracted. Second, a Gabor wavelet-based sclera pattern enhancement method, and an adaptive thresholding method to emphasize and binarize the sclera vein patterns has been implemented[1]. This increased the possibility to extract sclera vein pattern in a better way. The recognition is done using MATLAB. When the image matches from the data base it asks for selecting the bank when the user select the bank it ask the pin number of bank account and next processing starts like user can make transactions, check balance etc otherwise the process is cancelled. Sclera segmentation is the initial and the most challenging step in a sclera recognition system. The accuracy of the sclera recognition system could be degraded if the segmentation process fails to extract the correct sclera regions from an eye image. Some incorrect sclera segmentation scenarios include segmenting the sclera with some parts of the iris, eyelids, and eyelashes.

\section{LITERATURE SURVEY}

Sclera vein is a new biometric recognition method for human identification. Sclera vessel pattern's images are saturated and the arrangement of vessel patterns is multi layered and also it is quite complex to extract the features of vein from sclera[1]. The article reports and summarizes the results of competition on sclera segmentation and recognition benchmarking, called Sclera Segmentation and Recognition Benchmarking Competition 2016 (SSRBC 2016)[2]. Sclera vein recognition is shown to be a promising method for human identification. However, its matching speed is slow, which could impact its application for real-time applications, To improve the matching efficiency,a new parallel sclera vein recognition method is proposed using a two-stage parallel approach for registration and matching[3]. The contribution of this paper is the design of a robust sclera recognition system with high accuracy and the system comprises of new sclera segmentation and occluded eye detection methods[4].

\section{IMPLEMENTATION}

Take an Input image from the system. In preprocessing we convert our input colored image to grayscale. As we decode the whole thing in terms of binary. Applying horizontal sobel filter on that image, estimation of glare area, iris boundary

detection is done using circular iris segmentation method. Iris and eyelid detection and refinement is done using morphological operator and Gabor filter. 


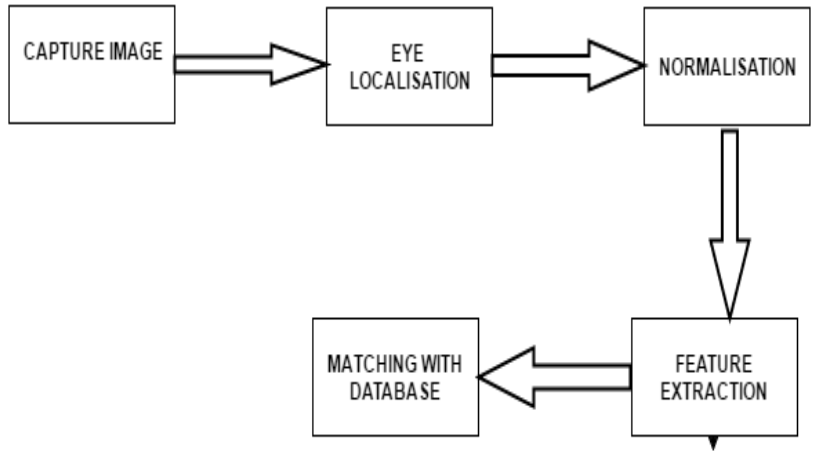

Fig 1. Block Diagram of Gabor Filter

\section{I - Scanned image \\ S - Sinusoidal wave \\ $\mathrm{F}$ - Frequency component \\ W-Wavelength \\ $\mathrm{P}-\mathrm{Phase}$ Component}

GABORFILTER( I,S,F,W,P) where

The 2D-Gabor filter is basically a bi-dimensional Gaussian function centered at origin $(0,0)$ with variance $S$ modulated by a complex sinusoid with polar frequency and phase. Wavelength of the sinusoidal carrier, specified as a numeric scalar in the range [2,Inf], in pixels/cycle. [mathworks]

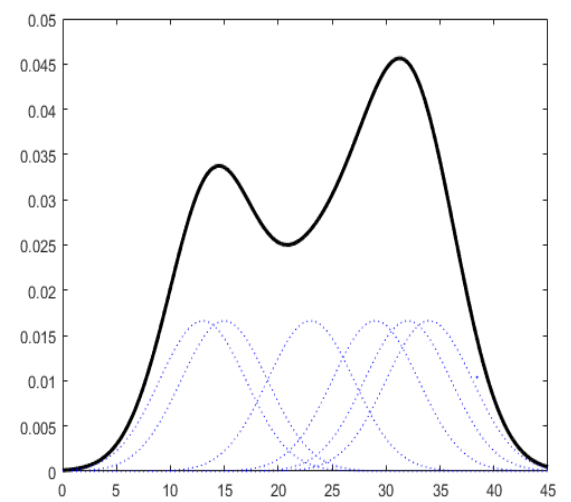

Fig .2. Kernel Function (Mathworks)

Kernel function is defined by a smoothing function and a bandwidth value that control the smoothness of the resulting density curve. It also defines the shape of the curve used to generate the probability density function. Smaller dashed curves are the probability distribution for each value of the sample data whereas larger solid curve is the overall kernel

distribution of the SIXMPG data. It is represented by an equation:

$f(x)=\frac{1}{\mathrm{nh}} \sum_{j=1}^{n} \mathrm{k}\left(\frac{\mathrm{x}-\mathrm{xj}}{\mathrm{h}}\right) \quad-\infty<\mathrm{x}<\infty$

(Mathworks)

If red plane is consideredcored for processing of sclera then the color of veins will be as same as that of color plane and thus system may not be able to differentiate between veins and plane[6]. Now if blue plane is used there are many people in European countries whose iris is blue in color, thus blue plane cannot be used for segmentation process. Thus it is advisable to use green plane as there are hardly few people in this world with green sclera. Sclera vein archetype are not readily visible under near infrared illumination, the normal illumination for iris perception algorithms, only iris vision databases that are assimilated under visible light illumination are potentially useful for sclera vein recognition applications. In this research, two databases are used - the UBIRIS databank and the IUPUI multiwavelength databank[7].The proposed sclera matching method uses a RANSAC-type registration algorithm to register the sclera vein descriptors, and the proposed sclera template matching method. The proposed sclera matching method is capable of matching the sclera vein patterns even in the presence of noise and deformations. Hamming distance is a distance measure for binary strings that measures the amount of similarity between two strings by measuring the number of bits that must be changed to make the two strings equivalent. In this model, it is used as the primary measure of how similar two features are. When acquiring the eye images, the eyelids can have distinct shapes, the iris location can differ, the pupil size can be different, and the eye may be tilted with respect to the camera[3]. The camera-to-object distance and camera zoom can also fluctuate. All of these factors could affect the size, the location, and the observed patterns of the acquired sclera region in the image. It is important to take these variances into account in a sclera matching algorithm. Therefore, the first step is to perform Sclera region-of-interest, or ROI, registration to achieve global rendition, revolution and scaling-invariance. In addition, due to the complex deformation that can occur in the vein archetype, it is desirable to have a registration scheme that is robust and exhaustive, but does not unduly introduce false accepts by over-fitting. The sclera vascular patterns deform non-linearly with the movement of the eye, eyelids, and the contraction/dilation of the pupil. As a result, the segments of the vascular patterns could move individually, and this must be accounted for in the registration scheme. To limit potential false accepts due to over fitting, the patterns are registered as a set of points - the centers of the line segments that make up the template. The optimal registration used is the one that minimizes the minimum distance between the templates. This reduces artificially introduced false accepts because it does not register the patterns using the same parameters used for matching, so the optimal registration and optimal matching can, and probably will, be different for templates that should not match. For the registration algorithm, it randomly chooses two points - one from the test template, and one from the target template. It also randomly chooses a scaling factor and a gyration value, based on prefetched knowledge of the database.

\section{OUTPUT}

\subsection{Normal Image}

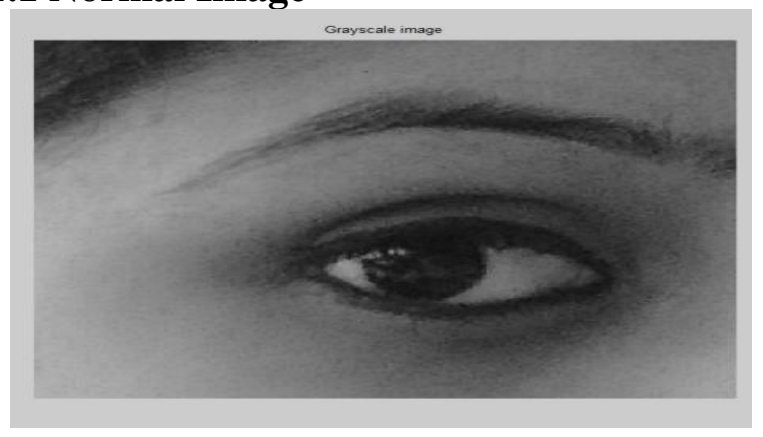

Fig 1.a. Grayscale image 


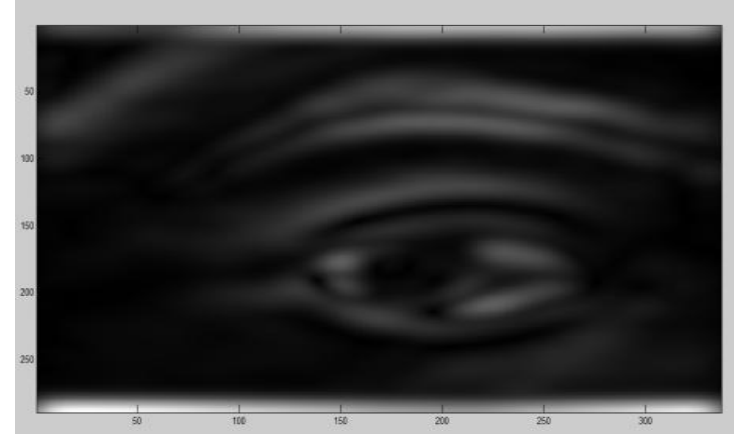

Fig 1.b.Magnitude of Grayscale image

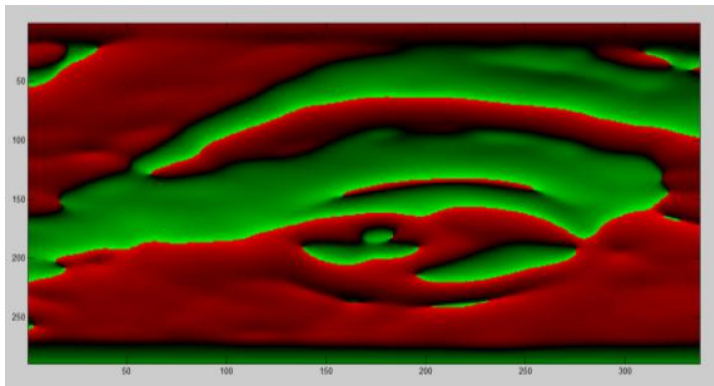

Fig 1.c. Phase of Grayscale image

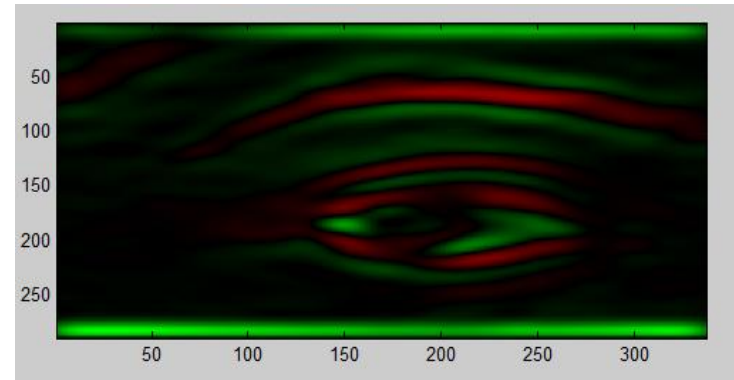

Fig 1.d. Colormap of real part

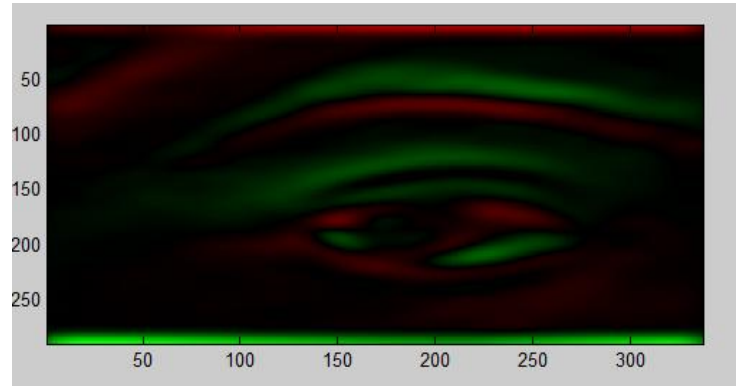

Fig 1.e Colormap of imaginary part

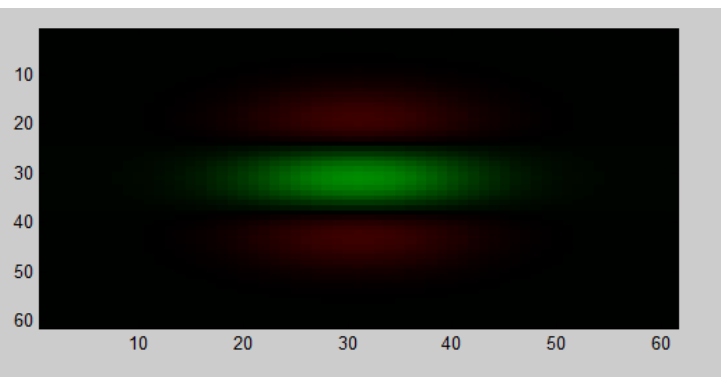

Fig 1.f Kernel of real part

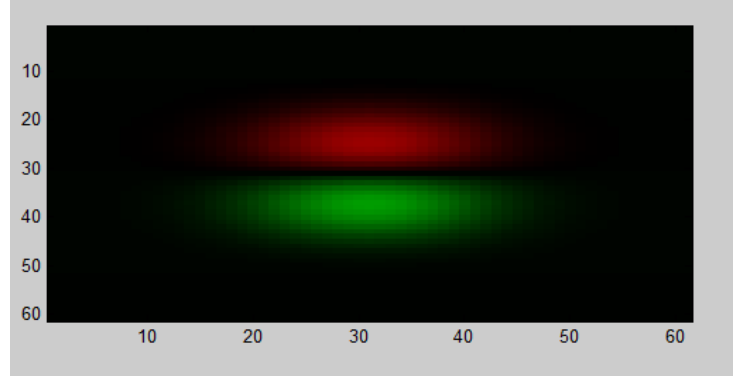

Fig 1.g. Kernel of imaginary part

\subsection{Image in Dark room}

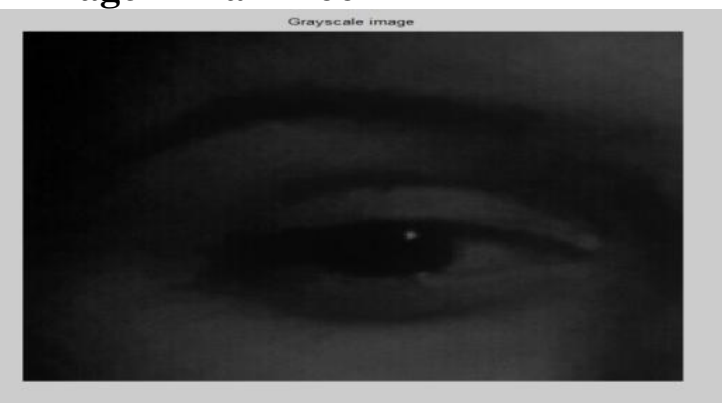

Fig 2.a Grayscale image in dark room

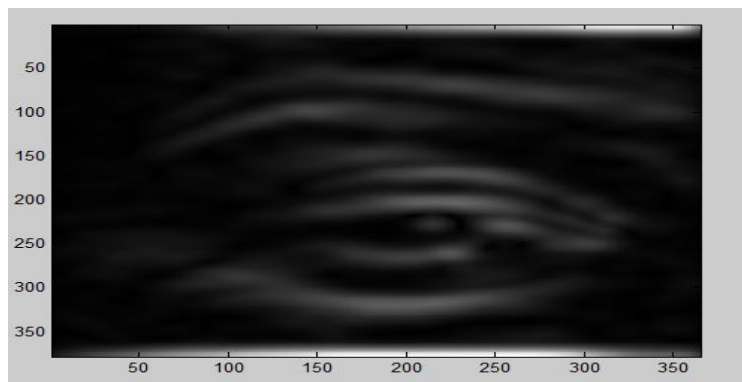

Fig 2.b Magnitude of image in dark room

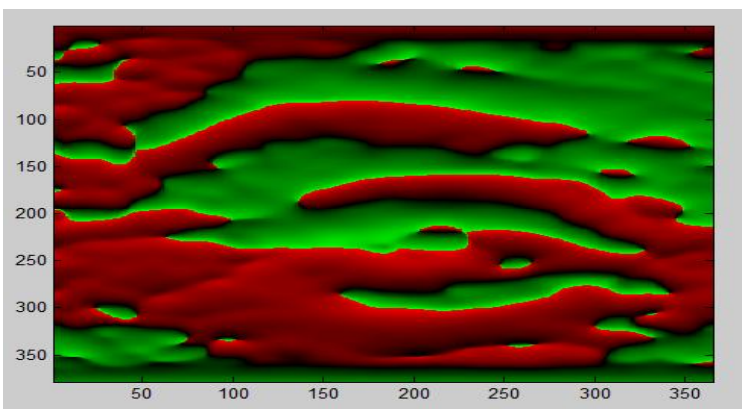

Fig 2.c Phase of image in dark room

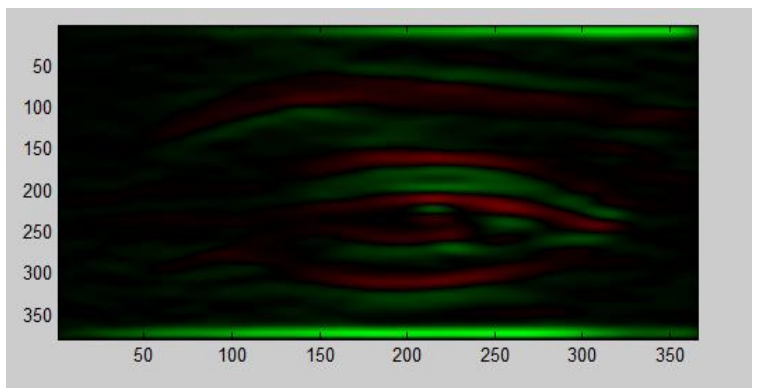

Fig 2.d. Colormap of real part 


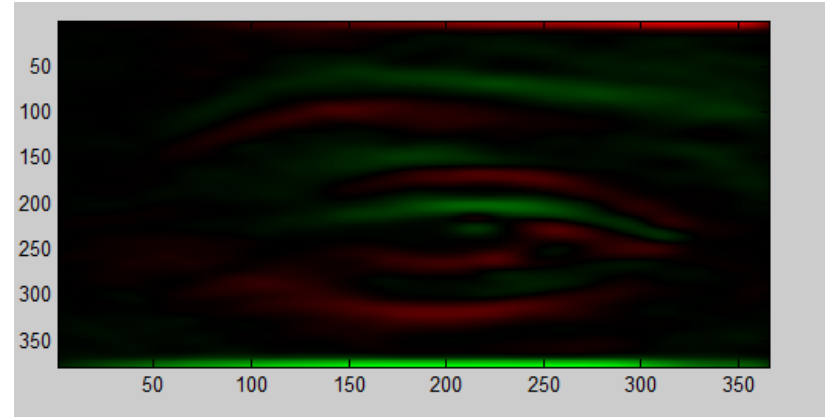

Fig 2.e Colormap of imaginary part of image in dark room

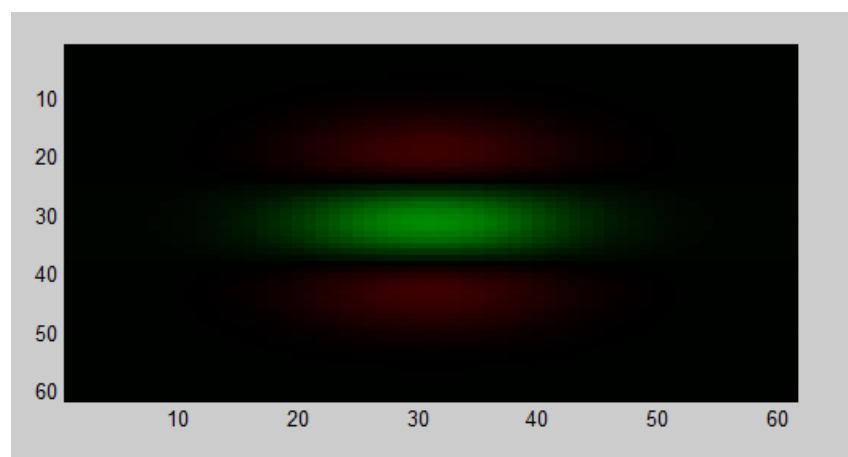

Fig 2.f Kernel of real part of image in dark room

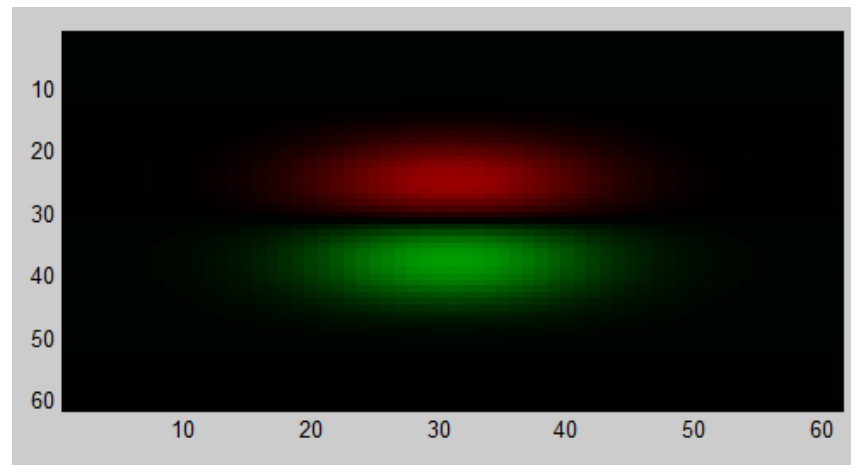

Fig 2.g. Kernel.of imaginary part of image in dark room

\subsection{Image under Flashlight}

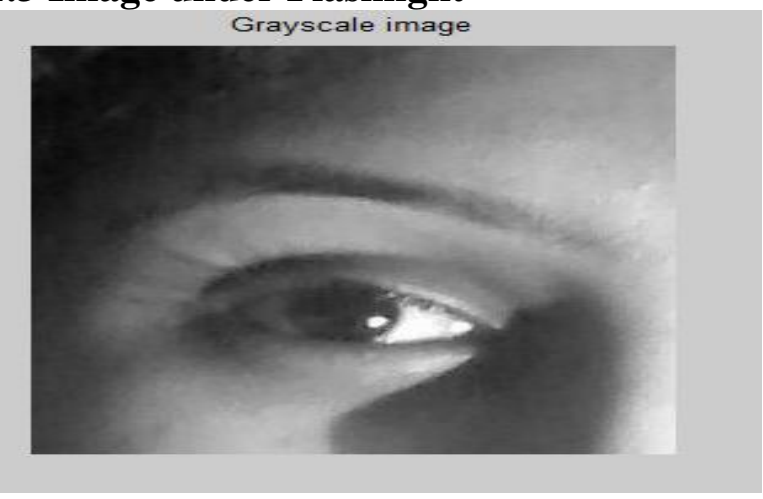

Fig 3.a. Grayscale image under flashlight

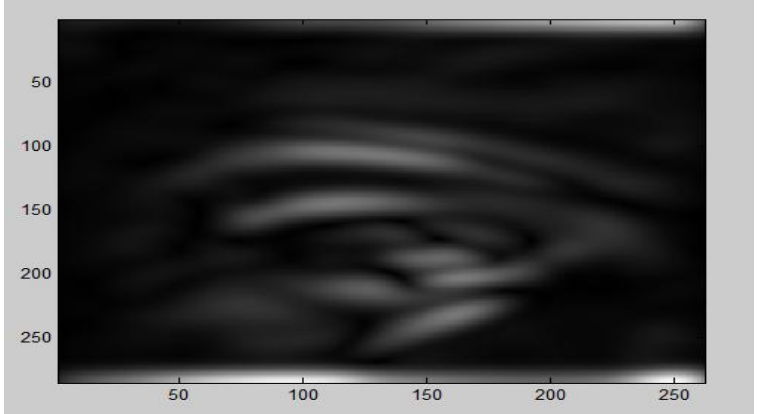

Fig 3.b Magnitude of image under flashlight

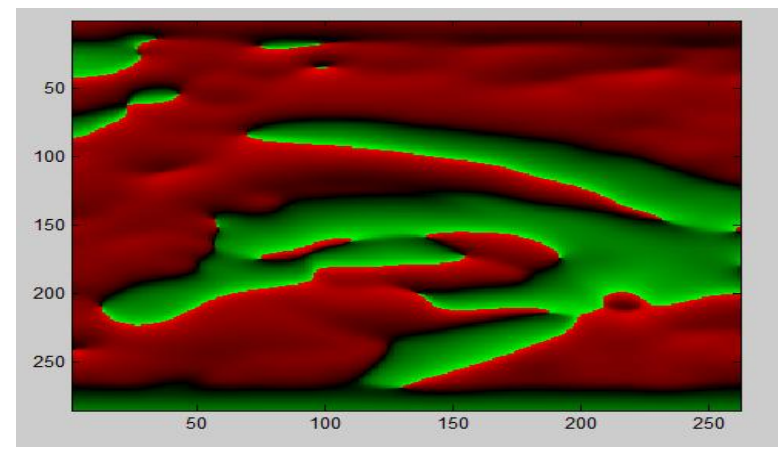

Fig 3.c. Phase of image under flashlight

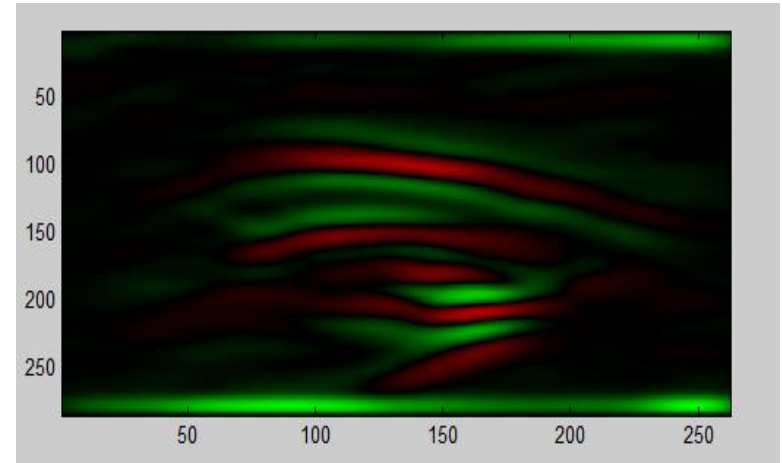

Fig 3.d Colormap of real part of image under flashlight

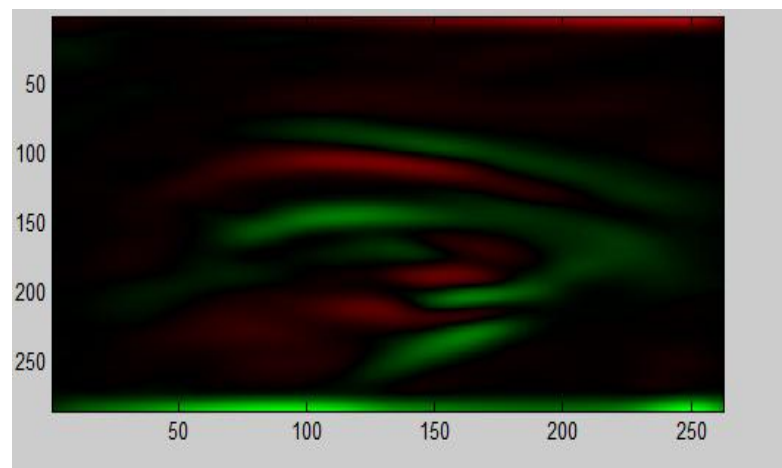

Fig 3.e. Colormap of imaginary part of eye under flashlight 


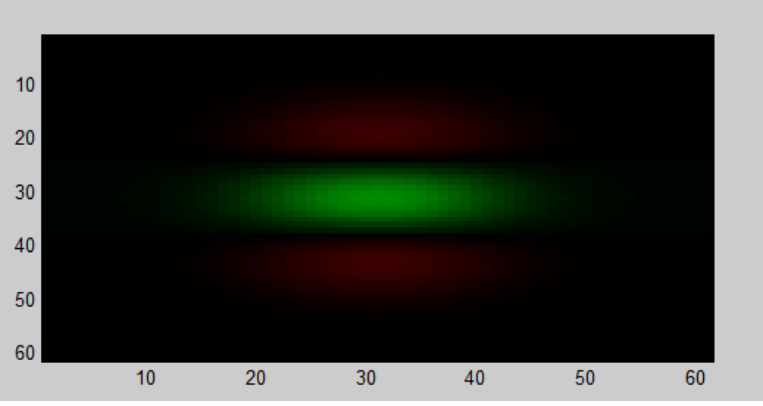

Fig 3.f. Kernel of real part of image under Flashlight

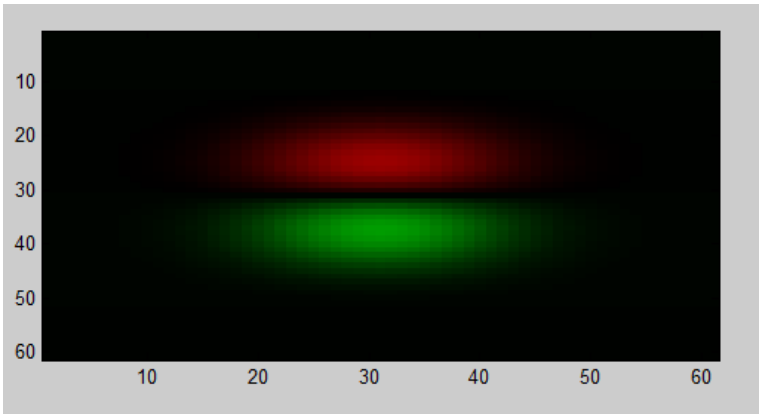

Fig 3.g. Kernel of imaginary part of image under flashlight

\section{CONCLUSION}

A new approach for automatic sclera segmentation, sclera pattern enhancement, sclera pattern template generation and sclera pattern matching is proposed. An automated sclera segmentation system that can work on both color and grayscale images and a novel line descriptor is proposed that can be used to accurately identify individuals from visible light

acquired. Sclera recognition overcomes all the drawbacks of iris, face as well as voice recognition.It is observed that when intensity of light increases colormap of image becomes more sharper. In contrary the image which is observed in a dark room has sclera patterns fader than the normal ones.

\section{FUTURE SCOPES}

Sclera recognition can be combined with other biometrics, such as iris recognition or face recognition (such as 2-D face recognition) to perform multimodal biometrics. Currently, the proposed system is implemented in Matlab. The processing speed can be dramatically reduced by parallel computing approaches.

\section{REFERENCES}

[1] A. Suganya (PG scholar) and M.Sivitha (Assistant Professor) ,"A New Biometric using Sclera Vein Recognition for Human Identification” in IEEE 2014.

[2] Abhijit Das, Umapada Pal, Miguel A. Ferrer and Michael Blumenstein,"SSRBC 2016: Sclera Segmentation and Recognition Benchmarking Competition” in IEEE 2016.

[3] Yong Lin, Eliza Yingzi Du, Senior Member, IEEE, Zhi Zhou, Student Member, IEEE, and N. Luke Thomas,"An Efficient Parallel Approach for Sclera Vein Recognition" in February 2014.

[4] S. Alkassar, Student Member, IEEE, W. L. Woo,Senior Member, IEEE, S. S. Dlay, and J. A. Chambers, Fellow, IEEE,"Robust Sclera Recognition System With Novel ScleraSegmentation and Validation Techniques" in IEEE 2015.

[5] S. Alkassar, W. L. Woo, S. S. Dlay and J. A.Chambers,"A novel method for sclera recognition with images captured on-the-move and at-adistance" in 2016 IEEE.

[6] S. Alkassar, W. L. Woo, S. S. Dlay and J. A. Chambers," Enhanced Segmentation and Complex- Sclera Features for Human Recognition with Unconstrained VisibleWavelength Imaging", in 2016 IEEE.

[7] Petru Radu, James Ferryman and Peter Wild,"A Robust Sclera Segmentation Algorithm", 1st in Sclera Segmentation Benchmarking Competition 2015.

[8] Abhijit Das, Umapada Pal, Miguel A. Ferrer and Michael Blumenstein ,"A new model sclera vessel recognition using OLBP", in springer international publishing Switzerland 2013.

[9] Parker, J.R., Duong A.Q," Gaze tracking: As sclera recognition approach", in 2009 IEEE (3836-3841). 